\title{
Tool wear monitoring using neuro-fuzzy techniques: a comparative study in a turning process
}

\author{
Agustin Gajate • Rodolfo Haber • Raul del Toro • \\ Pastora Vega * Andres Bustillo
}

\begin{abstract}
Tool wear detection is a key issue for tool condition monitoring. The maximization of useful tool life is frequently related with the optimization of machining processes. This paper presents two model-based approaches for tool wear monitoring on the basis of neuro-fuzzy techniques. The use of a neuro-fuzzy hybridization to design a tool wear monitoring system is aiming at exploiting the synergy of neural networks and fuzzy logic, by combining human reasoning with learning and connectionist structure. The turning process that is a well-known machining process is selected for this case study. A four-input (i.e., time, cutting forces, vibrations and acoustic emissions signals) single-output (tool wear rate) model is designed and implemented on the basis of three neuro-fuzzy approaches (inductive, transductive and evolving neuro-fuzzy systems). The tool wear model is then used for monitoring the turning process. The comparative study demonstrates that the transductive neuro-fuzzy model provides better error-based performance indices for detecting tool wear than the inductive neuro-fuzzy model and than the evolving neuro-fuzzy model.
\end{abstract}

Keywords Tool wear - Turning processes - Monitoring Neuro-fuzzy inference system · Transductive reasoning

\section{Introduction}

The strong international competition that exists in the field of manufacturing requires intelligent systems for providing greater value to the process and/or product (Rubio and Teti 2009). Machining processes play a crucial role in the field of manufacture and, therefore, it is an open field to deal with any kind of process improvement (Liang et al. 2004). Four basic types of machining operations are turning, drilling, milling, and grinding, which are performed by different machine tools. Indeed, the importance of maximizing a tool's working time and doing the utmost to keep tools from breaking is directly related to process optimization. The key issue is to find an appropriate trade-off between tool wear and productivity, considering the tool's cost, its replacement cost, the cost of writing off the machine's idle time, and so forth. Avoiding breakage derived from excessive tool wear is another important factor. The tool can be replaced after it breaks but it means increased costs, since the post-breakage stage is one of the trickiest, most unpredictable times, aside from the damage that may be done to the part and, not unusually, to the whole machine itself.

In the turning process, the tool is influenced by the combined action of large mechanical stress, high temperatures, and corrosion caused in part by cutting fluids. Thus, edges are gradually worn down and in extreme cases, it leads to premature catastrophic failure. Some important causes of tool breakage are plastic deformation, the material fluency at high temperature, and fatigue and brittle fracture because of combined stresses and low tenacity of the tool (Sharma et al. 2008b). For all these reasons, modeling, estimation and monitoring of tool wear are essential in any turning process (Rehorn et al. 2005).

However, due to the complexity of tool wear, its nonlinearity and the uncertainties of the process, there have 
been many approaches that have dealt with this problem through artificial intelligence techniques (Wang et al. 2008; Warnecke and Kluge 1998; Pal et al. 2009; Purushothaman 2009; Sharma et al. 2008a). Several of these approaches use artificial neural networks for modeling or monitoring tool wear in turning process (Sick 2002). The use of neural networks provides certain advantages, such as the capability of developing a model without requiring physical process knowledge. Nevertheless, this black box approach has a drawback because the model's structure is unable to offer any physical meaning. It means that it is not possible to extract information or knowledge from the created model (Sjoberg et al. 1995). In order to solve some of these drawbacks, the neuro-fuzzy systems emerge in the nineties combining the excellent ability to model any nonlinear function provided by neural networks and the semantic transparency provided by fuzzy logic (Jang 1993).

The simplest and easiest way to obtain a neuro-fuzzy model is to create its knowledge base using verbalization techniques. Frequently, however, a complete verbal description of how a complex process behaves is quite difficult to obtain. In such situations a procedure based on identification from input-output data is required. Therefore, a neuro-fuzzy model can be built from measured input/output (black box) data using engineering knowledge about the process variables, goals, and disturbances (white box) by applying recursive identifications techniques (error backpropagation, least squares, singular value decomposition, etc.).

The application of neuro-fuzzy system for modelling is not new and thousands of contributions have been reported in last decades. The complete review of the state-of-theart in this field goes beyond the scope of this paper. Some interesting approaches are recently reported in the literature (Cakmakci et al. 2010; Hayati et al. 2009; Perez et al. 2010; Sargolzaei and Kianifar 2010; Ubeyli 2009). Several neuro-fuzzy systems have been applied to optimize manufacturing processes through a new generation of model-based approaches. However, the use of neuro-fuzzy systems for modeling and monitoring tool wear is scarce (Abellan-Nebot and Romero Subiron 2010). In the case of turning processes, there are only few approaches based on the Adaptive Network Fuzzy Inference System (ANFIS) or in some variation of itself (Dinakaran et al. 2009; Li et al. 2000, 2004; Sharma et al. 2007, 2008a).

This paper presents two approaches for tool wear monitoring in turning processes based on neuro-fuzzy models. The main motivation is to perform a thorough comparative study to assess the suitability of state-of-art neurofuzzy strategies for tool wear monitoring. Previous works showed the first approximation using adaptive network-based fuzzy inference system (ANFIS) (Sharma et al. 2008a) and a transductive neuro-fuzzy system (TWNFIS) (Gajate et al. 2009). This work extends a preliminary comparative study (Gajate et al.
2009) by including another neurofuzzy system, a dynamic evolving neural-fuzzy inference system (DENFIS) (Kasabov and Song 2002), not previously reported. One contribution of this paper is a complete comparative study of neuro-fuzzy based model techniques applied to tool wear monitoring.

Moreover, in order to evaluate the suitability of the proposed strategy for tool wear monitoring, another material is used in the turning process. The change of material is considered a relevant disturbance for tool wear monitoring, and the three neuro-fuzzy systems are compared before this new situation. The main question is therefore whether the transductive neuro-fuzzy model achieves better error-based performance indices for detecting tool wear than other methods or not.

This paper is organized as follows: Section "Tool wear in turning process" describes the problem of tool wear in turning process; section "Neuro-fuzzy inference systems" presents the different neuro-fuzzy strategies to deal with the problem of tool wear monitoring; Section "Experimental setup" shows the experimental setup and the results of tool wear monitoring by the proposed systems. Finally, in section "Conclusions" the conclusions are showed.

\section{Tool wear in turning process}

Tool wear is generally caused by a combination of various phenomena, although it is an intrinsic event of cutting processes. Tool wear can occur gradually or in drastic breakdowns. Gradual wear may occur by adhesion, abrasion, or diffusion, and it may appear in two ways: wear on the tool's face or wear on its flank. Contact with the chip produces a crater in the tool's face. Flank wear, on the other hand, is commonly due to friction between the tool and the workpiece material.

The importance of maximising a tool's working time and doing the utmost to keep tools from breaking is directly related with cutting-process optimisation. One of the main goals in turning (as in other machining processes) is to achieve an economic tool-life through wisely chosen cutting speeds, cutting feeds and depths of cut. The key issue is to find an appropiate trade-off between tool wear and productivity considering the tool's cost, its replacement cost, the cost of writing off the machine's idle time, and so forth. Avoiding breakage is another capital factor, because replacing the tool after it breaks means increased costs, since the post-breakage stage is one of the trickiest, most unpredictable times, aside from the damage that might be done to the part and, not unusually, to the whole machine itself.

Tool wear is not a physical variable value which is easily measured by some specific method, but rather a subjective estimate a specialist can make, depending on the condition of the tool's edges and surfaces. Since there is no single criterion for deciding when a tool needs sharpening, different lifetimes may be predicted for the same tool employed in 


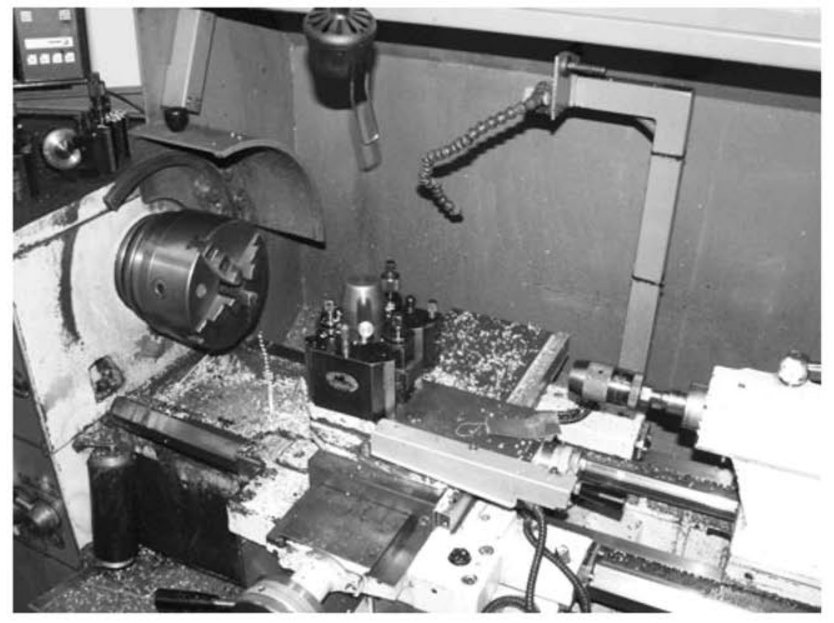

Fig. 1 Lathe machine in detail

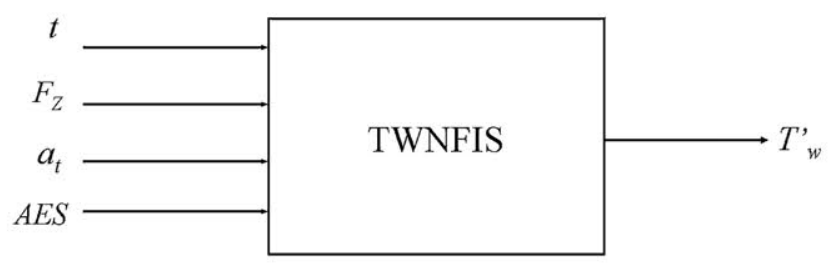

Fig. 2 Tool wear model

the same process. Two widely used criteria are catastrophic failure and changes in tool geometry. Other criteria that are sometimes used are a degraded tool-surface finish, deviation in cutting forces, increased power consumption, overheating, non-tolerant pieces, and the appearance of chattering. This paper deals with the tool wear modeling of the turning process (Fig. 1). The ultimate goal is to implement the developed model in a process for monitoring on-line the tool wear in a turning process. Due to this objective, it is interesting to know what is exactly happening in the process through process signals that provide the most information about the tool wear process and therefore, to monitoring the process itself.

In order to be consistent with the state of the art, inputoutput data measured from sensoring signals such as acoustic emissions signals $(A E S)$, vibrations (accelerations) $\left(a_{t}\right)$, cutting forces $\left(F_{z}\right)$ and time $(t)$ (Fig. 2) are used as model inputs to estimate tool wear (Sharma et al. 2008a). Therefore, a multiple-input/single-output model is designed and implemented for monitoring tool wear.

The cutting force, vibration, time, and acoustic emission variables are selected because quite often the tool wear phenomenon is reflected by time-domain and frequency domain analysis of these variables. The acoustic emission can be used to detect gradual and abrupt tool wear. Cutting tool vibrations during machining are produced due to rubbing action at the work-piece tool flank interface, formation of built-up edge, and waviness of the work surface. Cutting force is a variable that is relatively easy to be measured in real-time.
The tangential component of force $\left(F_{z}\right)$ is more sensitive to tool wear as compared to axial component $\left(F_{x}\right)$ and radial component $\left(F_{y}\right)$. The literature in the field of machining processes (and turning processes) reflects this fact (Dimla 2000). Moreover, the value $F_{z}$ determines the torque on the main drive mechanism, the deflection of the tool, and the required power.

\section{Neuro-fuzzy inference systems}

Neuro-fuzzy inference techniques combine the paradigms of fuzzy logic and neural networks in order to take advantage of both techniques, achieving the simplicity of modeling (neural networks), while providing knowledge explicitly expressed in a set of if-then rules. Neuro-fuzzy systems have been widely used in modeling, identification and monitoring of complex systems. Since its origin in the early nineties, neuro-fuzzy systems have undergone various changes over the years, giving rise to various trends in research. For example, depending on the type of inference that the neurofuzzy system uses, or according to the structure of the neurofuzzy system, it can distinguish various sub-groups within the neuro-fuzzy approaches.

In terms of learning procedures (type of inference), most evolutionary neuro-fuzzy strategies apply inductive reasoning systems. In inductive reasoning the key issue is to find a general model (function) drawn from the entire set of input/output data representing the whole system. The model is later used for designing the required control system. In contrast, there are transductive reasoning methods that generate a model at a single point of the workspace, giving rise to transductive neuro-fuzzy inference systems.

According to the structure, an evolving neuro-fuzzy system is able to update its knowledge and refine the model through interaction with the environment. The main advantage in the use of these systems for modeling and monitoring process is that the structure of the evolving neuro-fuzzy system changes depending on what the process demands, unlike the current neuro-fuzzy systems which have fixed structure.

Transductive methods have some advantages over inductive methods, because sometimes creating a valid model for the entire space or region of operation is a difficult task, yielding inadequate performance in some cases. The dynamic generation of local models enables the knowledge represented as the set of known data facilitating incremental on-line learning to be expanded easily. In addition, these strategies are capable of functioning correctly with a small training set.

Based on the above definitions, this paper presents three neuro-fuzzy systems that combine these characteristics. On the one hand we have the neuro-fuzzy system par excellence: ANFIS. It has inductive inference and fixed structure. On the one hand, it has showed a Dynamic Evolving Neural-Fuzzy 
Fig. 3 ANFIS architecture

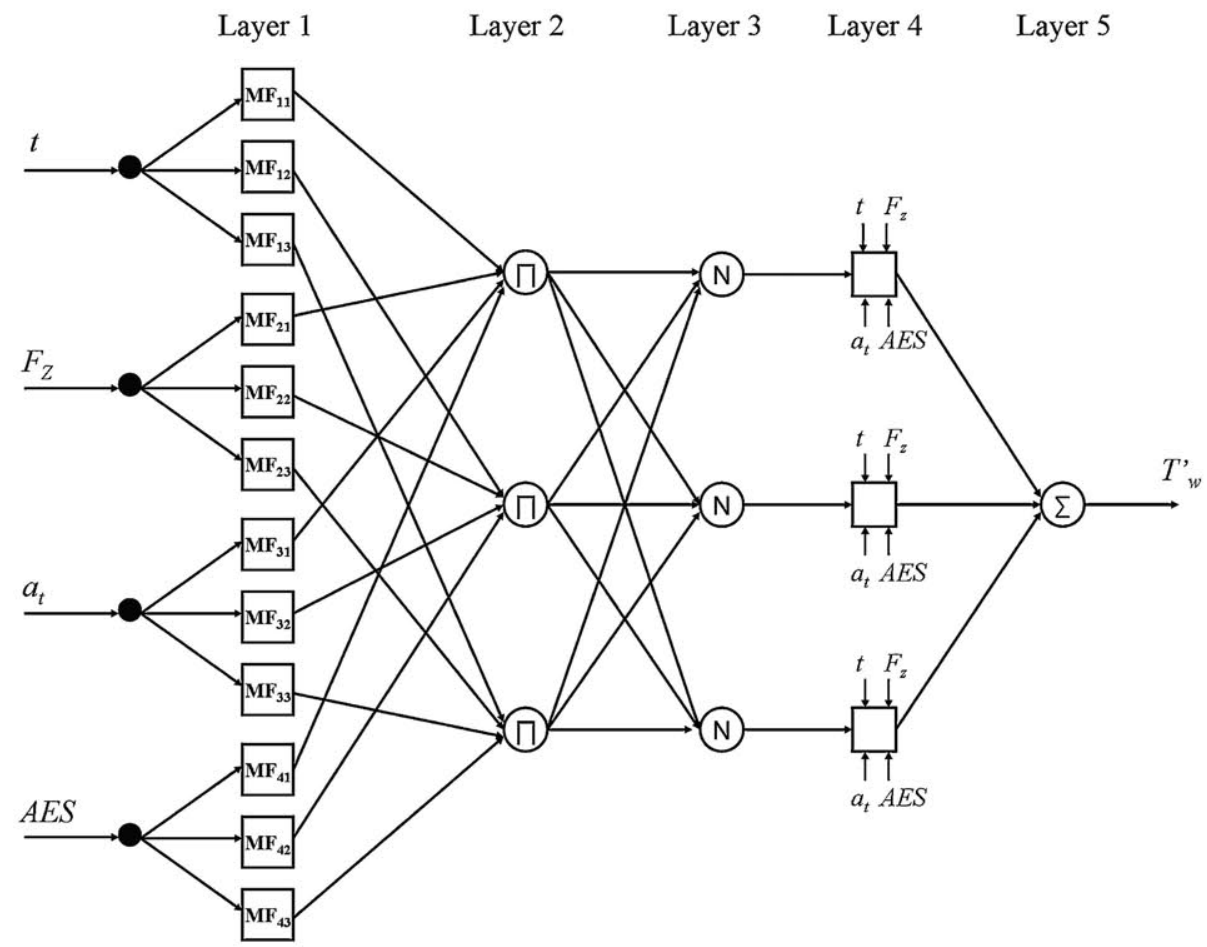

Inference System (DENFIS), which is considered one of the first systems with the evolving and neuro-fuzzy principles. It has inductive inference. Finally it has presented a Transductive-Weighted Neuro-Fuzzy Inference System (TWNFIS), which is a relatively new transductive reasoning system that consists of a dynamic neuro-fuzzy inference system with local generalization. It is also considered an evolving neurofuzzy systems because its structure changes over the time.

\section{Adaptive-network-based fuzzy inference system (ANFIS)}

ANFIS system is one of the first neuro-fuzzy systems to be developed (Jang 1993). Its principle is based on extracting fuzzy rules in each level of a neural network. Once the rules have been obtained, they provide the necessary information on the global behavior of the system.

ANFIS implements the Takagi-Sugeno model. The ANFIS architecture has five layers, as shown in Fig. 3. The nodes represented with squares are nodes with adjustable parameters, whereas the nodes represented by circles are fixed nodes. The first layer represents fuzzy membership functions. The second and the third layer contain nodes that form the antecedent parts in each rule. The fourth layer calculates the first-order Takagi-Sugeno rules for each fuzzy rule. The fifth layer (output layer) calculates the weighted global output of the system.

ANFIS uses error back propagation as the learning strategy to determine the antecedent parameters of the rules. The consequent parameters of each rule are determinated using the least squares method. A step in the learning procedure has got two passes: in the first or forward pass, the input patterns are propagated, and the optimal consequent parameters are estimated by an iterative least mean square procedure, while the premise parameters are assumed to be fixed for the current cycle through the training set. In the second or backward pass the patterns are propagated again, and in this epoch, back propagation is used to modify the premise parameters, while the consequent parameters remain fixed. This procedure is then iterated until the error criterion is satisfied (Denai et al. 2007).

Dynamic evolving neural-fuzzy inference system (DENFIS)

The Dynamic Evolving Neural-Fuzzy Inference System (DENFIS) is an application of the ECoS principles to an ANN that implements a Takagi-Sugeno fuzzy inference system and triangular membership functions (Watts 2009). DENFIS is thoroughly described in Kasabov and Song (2002).

DENFIS utilizes a dynamic inference for adaptive online and offline learning. DENFIS evolves through incremental learning and accommodate new input data including new features through local element tuning. New fuzzy rules are created and updated during the operation of the system. At each time moment, the output of DENFIS is calculated through a fuzzy inference system based on $m$-most activated fuzzy rules which are dynamically chosen from a fuzzy rule set. A set of fuzzy rules can be inserted into DENFIS before or during its learning process. Fuzzy rules can also be extracted during or after the evolving process. 
DENFIS use the so-called Evolving Clustering Method (ECM) (Song and Kasasbov 2001). This is based on the concept of dynamically adding and modifying the clusters as new data are presented, where the modification to the clusters affects both the position of the clusters and the size of the cluster, in terms of a radius parameter associated with each cluster that determines the boundaries of that cluster. ECM has only one parameter, which drives the addition of clusters, known as the distance threshold $D_{t h r}$. When new clusters are added, their centers are set to equal the example that triggered their creation, and the radius $R$ of a new cluster is initially set to zero. $R$ grows as more vectors are allocated to the cluster. Due to the mechanism by which $R$ is updated, it cannot exceed $D_{t h r}$.

The main ECM function is to support the inference of fuzzy rules from data in DENFIS. This is done in two phases, first forming the antecedents, followed by the consequent functions. The antecedents are formulated by finding which combination of input Membership Functions (MFs) activates most highly for the center of the cluster, i.e., the values represented by the cluster center are fuzzified by the input MF set and the winning, most highly activated, MFs are taken as the antecedents for that rule.

The consequent functions are then calculated using a least means estimation process over the examples within the cluster. Thus, each cluster is used as the basis of a single rule. Clustering and reformulation of the rules are performed whenever a new training example is presented to the network. For any input vector, the output of the DENFIS in calculated as the combined output of the most strongly activated $m$ rules. There is no adjustment of the MF during training.

Transductive-weighted neuro-fuzzy inference system (TWNFIS)

The Transductive-Weighted Neuro-Fuzzy Inference System (TWNFIS) is a relatively new transductive reasoning system that consists of a dynamic neuro-fuzzy inference system with local generalization (Song and Kasabov 2006). TWNFIS is endowed with three important characteristics:

- Neural: Excellent ability to model any nonlinear function with a high accuracy in addition to possessing a high learning capacity.

- Fuzzy: Semantic transparency, ability to represent human thought as well as excellent behavior when there is uncertainty and imprecision.

- Transductive: Estimation of the model in a single input/ output set of the space, using only information related with the corresponding set.

In this work it has used a modification of the original work (Song and Kasabov 2006) that was already shown in Gajate

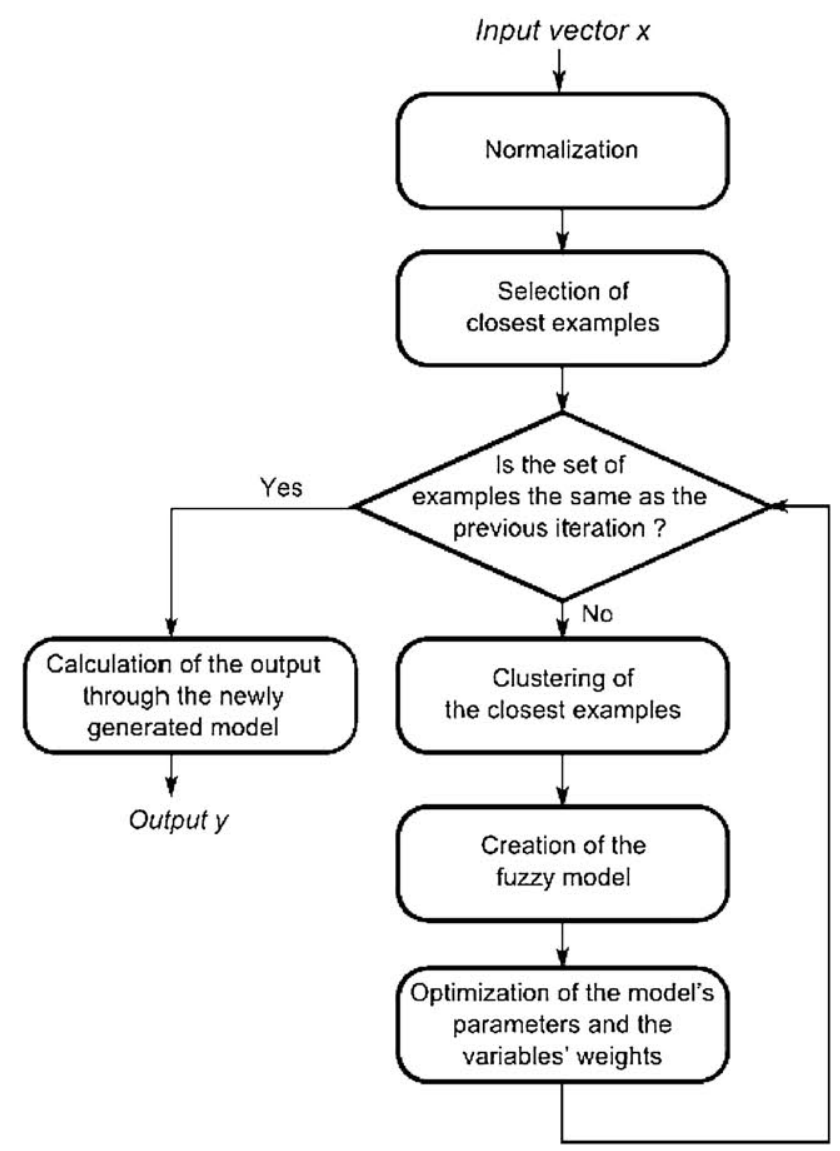

Fig. 4 Diagram of the transductive neuro-fuzzy inference system algorithm

et al. (2009). The modified TWNFIS algorithm differs in two main issues from the original algorithm: normalization data procedure and clustering algorithm. The normalization procedure is based on mean and standard deviation of the overall data set unlike the max-min normalization reported in Song and Kasabov (2006). Quality threshold (QTCA) clustering is applied instead of the Evolving Clustering Method (ECM) suggested by the same authors. Quality is ensured in QT clustering by finding large clusters whose diameter does not exceed a given user-defined diameter threshold. This method prevents that dissimilar data is included in the same cluster and it ensures that only good quality clusters will be created. Gaussian membership functions and Mamdani-type systems are used in both approaches. Figure 4 shows the different steps of the proposed approach to create each local neurofuzzy model. The explanations of each step are given as follows.

Firstly, the model's inputs in each sample time (e.g., time, acceleration, force) can be treated in different kinds of physics units but normalization is recommended. In this paper, each input data $x^{\prime}$ is normalized according to (1):

$x=\frac{x^{\prime}-\mu_{x}}{\sigma_{x}}$ 
where $\mu_{x}$ is the mean and $\sigma_{x}$ is the standard deviation of the set of known data or training set.

The different local models are created using data from the training set that are the closest to each new input datum. The weighted Euclidean distance is used for selecting each data subset (2), in other words, the nearest neighbors. The size of the subset of neighbors $\left(N_{q}\right)$ is one parameter of the algorithm. Weights $\left(w_{j}\right)$ of each element of the input vector $\left(w_{j} \in[0,1]\right)$ are computed in an a posteriori model-adjusting process, reflecting the importance of each variable. Initially they all have unitary value. Once it has computed the nearest neighbors, the algorithm calculates the weights for each calculated distance (3).

$$
\begin{aligned}
\|\bar{x}-\bar{y}\| & =\left[\frac{1}{P} \sum_{j=1}^{P} w_{j}\left|x_{j}-y_{j}\right|^{2}\right]^{\frac{1}{2}} \\
v_{i} & =1-\left(d_{i}-\min (\bar{d})\right)
\end{aligned}
$$

where $P$ is the number of elements in the input data vector, $\bar{x}$ is the input data vector, $\bar{y}$ is each of the vectors in the training set, $\min (\bar{d})$ is the minimum element in the distance vector $\bar{d}=\left[d_{1}, d_{2}, \ldots, d_{N_{q}}\right]$, and $i=1,2, \ldots, N_{q}$ is the index representing the number of closest neighbors.

When the subset has been chosen and the distance weights have been calculated, fuzzy rules and membership functions (with their initial parameters value) are built iteratively on the basis of the closest data. The next step of TWNFIS is the use of the Evolving Clustering Method (ECM) (Kasabov and Song 2002) to create these neuro-fuzzy local models. The main difference with regard to the approach proposed herein is the use of a clustering algorithm more suitable for real-time modeling of dynamic systems instead of ECM. A clustering strategy called the Quality Cluster Algorithm (QTCA) is then applied (Heyer et al. 1999). This algorithm utilizes two parameters: a threshold to indicate a maximum diameter of the clusters and a minimum number of elements (data) in a cluster. A candidate cluster is created using the first datum. The other elements are iteratively added without exceeding the maximum diameter. A second candidate cluster is formed starting with the second datum and repeating the procedure. The number of candidate clusters is equal to the number of closest data. At this point, the largest candidate cluster is selected and retained. Data are removed from consideration and the entire procedure is repeated on the smaller set. A stopping criterion is when the largest remaining cluster has fewer elements than a pre-specified number of elements. The resulting clusters are ellipsoids. The center and the radius of the clusters set the center and width of the Gaussian membership functions, respectively. Each fuzzy rule is created based on a cluster.
Considering $P$ inputs, one output and $M$ fuzzy rules initially defined by the clustering algorithm, the $l$ th rule has the form:

$R_{l}$ : If $x_{l}$ is $\phi_{l 1}$ and $x_{2}$ is $\phi_{l 2}$ and... $x_{P}$ is $\phi_{l P}$, then $y$ is $\gamma_{l}$. (Cluster $l$ )

$$
\begin{aligned}
\phi_{l j} & =\alpha_{l j} \exp \left[-\frac{\left(x_{j}-m_{l j}\right)^{2}}{2 a_{l j}^{2}}\right] \\
\gamma_{l} & =\exp \left[-\frac{\left(y-n_{l}\right)^{2}}{2 \delta_{l}^{2}}\right]
\end{aligned}
$$

where $m$ and $n$ are the centers of the Gaussian functions for the inputs and outputs, $a$ and $\delta$ are the widths, $i=$ $1,2, \ldots, N_{q}$ is the index representing the number of closest neighbors, $j=1,2, \ldots, P$ represents the number of input variables, and $l=1,2, \ldots, M$ represents the number of fuzzy rules.

Defuzzification is carried out using a modified center of area method. The resulting error function is stated as a weighted quadratic error function that is derivable:

$$
\begin{aligned}
f\left(\bar{x}_{i}\right) & =\frac{\sum_{l=1}^{M} \frac{n_{l}}{\delta_{l}{ }^{2}} \prod_{j=1}^{P} \alpha_{l j} \cdot \exp \left[-\frac{w_{j}{ }^{2}\left(x_{i j}-m_{l j}\right)^{2}}{2 a_{l j}^{2}}\right]}{\sum_{l=1}^{M} \frac{1}{\delta_{l}{ }^{2}} \prod_{j=1}^{P} \alpha_{l j} \cdot \exp \left[-\frac{w_{j}{ }^{2}\left(x_{i j}-m_{l j}\right)^{2}}{2 a_{l j}^{2}}\right]} \\
E & =\frac{1}{2} v_{i}\left[f\left(\bar{x}_{i}\right)-q_{i}\right]^{2}
\end{aligned}
$$

where $f\left(x_{i}\right)$ is the defuzzification function that yields the output, $q_{i}$ are the target values, and $v_{i}$ indicates the distance weight (the proximity of each target to the expected prediction).

The weight of each variable is adjusted according to its relevance within each sub-space. A gradient-descent algorithm to optimize the weights and parameters of the fuzzy rules is then applied after deriving (7). If the closest neighbors do not change due to the new adjusted weights of the variables, a new model is created setting the weights obtained in the previous iteration. Finally, the model is used to predict the system output (Fig. 5). A more in-depth explanation of the different steps made can be found in Song and Kasabov (2006).

\section{Experimental setup}

Monitoring of tool wear for turning processes is based on the experimental setup and the experimental data presented in Sharma et al. (2008a) and (2007).

The main rationale of using the experimental data obtained by Sharma et al. (2008a) and (2007) is to propose a modelbased method inspired by emerging neuro-fuzzy approaches 
Fig. 5 TWNFIS architecture in an example of creation of 3 clusters (the connection between layer 1 and layer 2 depends on the clusters created)

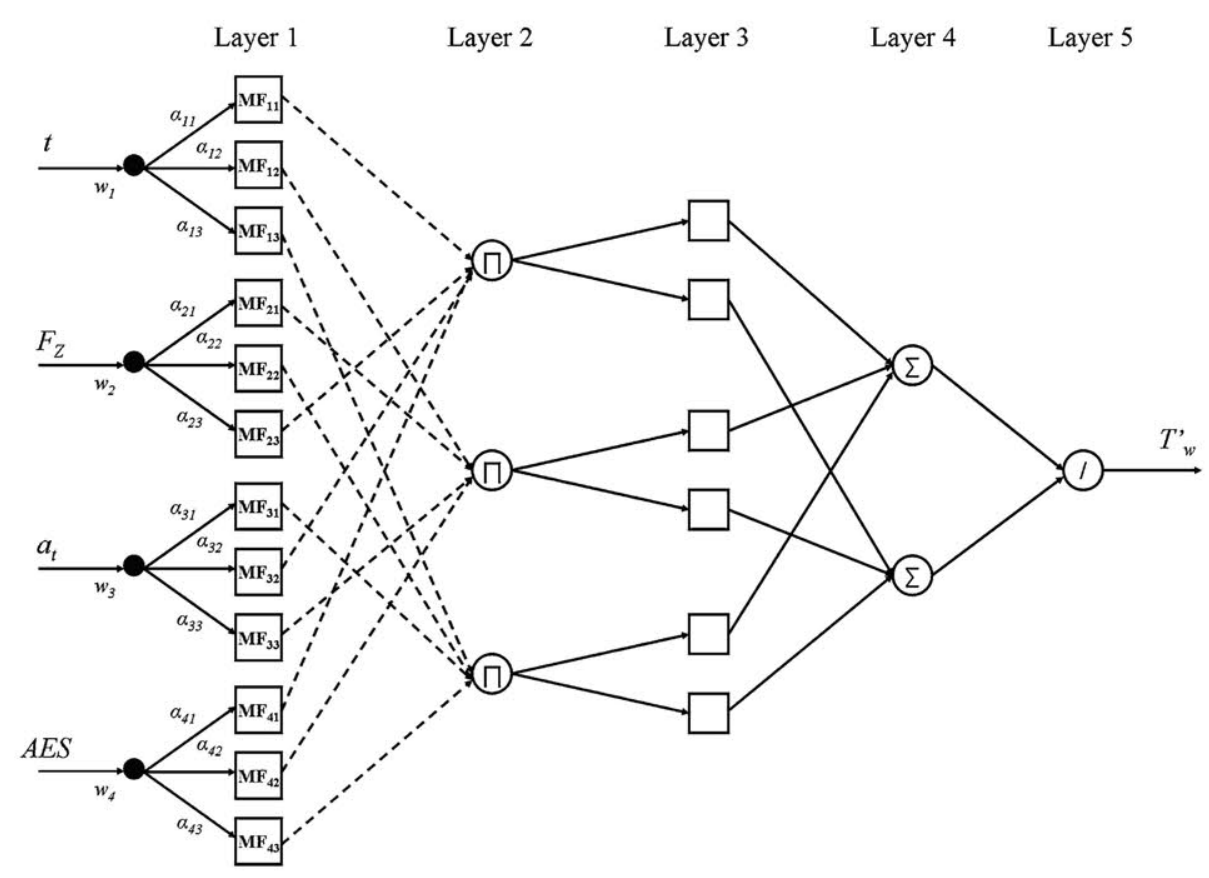

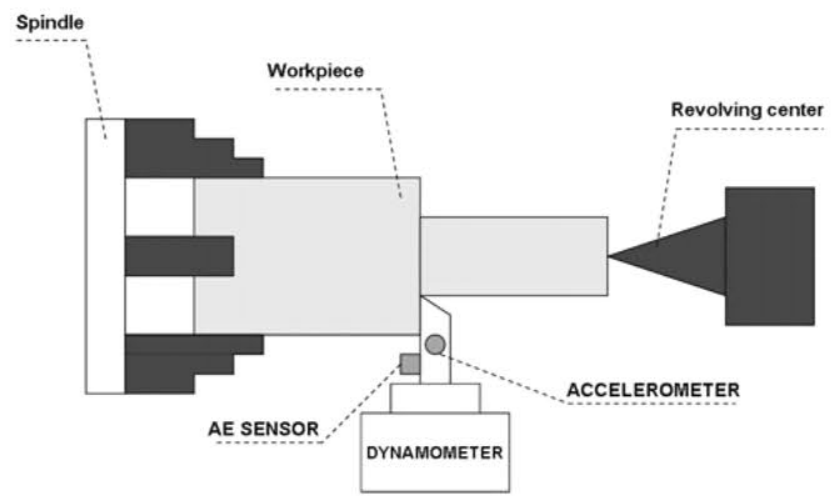

Fig. 6 Experimental setup

that can be easily verified on the basis of already presented results. Indeed, it is quite common in many fields to use databases to corroborate (or not) the suggested approaches of researchers. Moreover, the suitability of the suggested approaches using experimental data already reported are the best basement for performing further comparative studies. It means that comparative studies and the results can be really useful from a practical viewpoint.

The experimental setup is shown in Fig. 6. Acoustic emission signals (ring down count), vibrations (acceleration), cutting forces, time and tool wear were recorded for each operation on the machine. A brief description of this experimental setup is given as follows.

The turning operations were carried out on a high precision lathe machine. The conventional tool post of the lathe machine is removed and the tool dynamometer is fixed in its place. Then the tool is mounted in the tool dynamometer slot. For measuring acceleration, the accelerometerć6s prod is placed on the top surface of the tool. The AE Sensor (piezoelectric transducer) is fixed on the tool holder using a layer of couplant. The pencil lead break test was used to calibrate $\mathrm{AE}$ to estimate the attenuation factor of the $\mathrm{AE}$ signal when the signal was transmitted from the work piece to the cutting tool. The detected signals were amplified and filtered through band-pass filter. The conditional signals were recorded in the computer for further analysis. Cutting fluid was not used during the cutting process. Tool wear (flank wear) was measured off-line using a microscope.

Experimental data were obtained from turning operations on two different workpiece materials: cast iron (grey cast iron-FG 15) and an alloy steel (En 24). In this way, it will be tested the validity of the model for different materials. For turning operations in both materials, an uncoated carbide insert tool material (CCMT 060204 TTS) was used. The tool holder was a SCLCR 1010E06 (WIDIA make).

For each material were carried out four experiments. The process parameters were the same for both materials. Turning operations were conducted at two cutting speeds: $94 \mathrm{~m} / \mathrm{min}$ and $188 \mathrm{~m} / \mathrm{min}$. For each speed were selected two cutting feeds: $0.06 \mathrm{~mm} / \mathrm{rev}$ and $0.08 \mathrm{~mm} / \mathrm{rev}$. The depth of cut was kept constant for all operations and in both materials $(0.7$ $\mathrm{mm}$ ). In total, eight experiments were conducted (Table 1) whose results are reflected in the appendix of this work.

\section{Results}

The tool wear $T_{w}^{\prime}$ was modeled through time $t$, the cutting force in the direction of cutting speed $F_{z}$, the vibrations (accelerations) of the tool $a_{t}$, and the acoustic emission 
Table 1 Experiments

\begin{tabular}{llllll}
\hline $\begin{array}{l}\text { Experiment } \\
\text { number }\end{array}$ & $\begin{array}{l}\text { Worpiece } \\
\text { material }\end{array}$ & $\begin{array}{l}\text { Cutting } \\
\text { speed }(\mathrm{m} / \mathrm{min})\end{array}$ & $\begin{array}{l}\text { Cutting } \\
\text { feed }(\mathrm{mm} / \mathrm{rev})\end{array}$ & $\begin{array}{l}\text { Depth } \\
\text { of cut }(\mathrm{mm})\end{array}$ & Tool material \\
\hline 1 & FG 15 & 94 & 0.06 & 0.7 & Uncoated carbide CCMT 060204 TTS \\
2 & FG 15 & 94 & 0.08 & 0.7 & Uncoated carbide CCMT 060204 TTS \\
3 & FG 15 & 188 & 0.06 & 0.7 & Uncoated carbide CCMT 060204 TTS \\
4 & FG 15 & 188 & 0.08 & 0.7 & Uncoated carbide CCMT 060204 TTS \\
5 & En 24 & 94 & 0.06 & 0.7 & Uncoated carbide CCMT 060204 TTS \\
6 & En 24 & 94 & 0.08 & 0.7 & Uncoated carbide CCMT 060204 TTS \\
7 & En 24 & 188 & 0.06 & 0.7 & Uncoated carbide CCMT 060204 TTS \\
8 & En 24 & 188 & 0.08 & 0.7 & Uncoated carbide CCMT 060204 TTS \\
\hline
\end{tabular}

signals $A E S$ in order to monitor the process. The use of these four signals is because all of them provide relevant information about the tool wear. The elimination of some of them greatly deteriorates the process monitoring. Therefore, depending on the model, the tool wear was estimated as follows:

$T_{w}^{\prime}=\hat{H}\left(t, F_{z}, a_{t}, A E S\right)$

where $\hat{H}$ represents the corresponding neuro-fuzzy system.

The total average error $T A E$ (9) is used to assess the accuracy of the models. Another measure of accuracy is the number of data (in each experiment) that surpasses an average error of $10 \%$. This figure of merit is chosen because at the industry level (especially dealing with processes' monitoring) certain margins of error are generally acceptable due to signal noise and a certain inaccuracy of the sensors. In this sense, all error less than $10 \%$ is usually more or less acceptable (depending on individual cases), whereas when dealing with errors greater than $10 \%$, it has to be careful with that information.
$T A E=\frac{1}{n} \sum_{n} \frac{\left|T_{w}-T_{w}^{\prime}\right|}{T_{w}} \cdot 100$

where $T_{w}$ is the real tool wear, $T_{w}^{\prime}$ is the modeled tool wear, and $n$ is the number of data of each experiment.

The parameters of the neuro-fuzzy systems that best match the model with the process after testing several configurations are summarized in Table 2. The parameters of the neurofuzzy models are the same for both turning operations with FG 15 workpiece material, as for turning operations with En 24, excepting the number of membership functions in ANFIS (and therefore, the number of rules). Moreover, in training data sets, it has only been used data of the material to be machined.

ANFIS parameters have been chosen in accordance to the reported in Sharma et al. (2007, 2008a). TWNFIS parameters are maintained with respect to Gajate et al. (2009) unless the clustering threshold of QTCA. In this case, it has made a thoroughly study about a more appropriate clustering threshold obtained as a result a clustering threshold

Table 2 Neuro-fuzzy algorithms for modeling tool wear

\begin{tabular}{llll}
\hline Algorithm & ANFIS & DENFIS & TWNFIS \\
\hline System & MISO & MISO & MISO \\
Clustering & Substractive & ECM algorithm & Quality algorithm \\
Structure & Fixed & Variable & Variable \\
Inference & Inductive & Inductive & Transductive \\
Membership functions (MFs) type & Gaussian & Triangular & Gaussian \\
Inference system & Takagi-Sugeno & Takagi-Sugeno & Mamdani \\
Number of MFs (FG 15/En 24) & $7 / 10$ & 4 & Variable each run (max. 4) \\
Number of rules (FG 15/En 24) & $7 / 10$ & 4 & Variable each run (max. 4) \\
Training algorithms & Back propagation \& least squares method & Least squares method & Back propagation \\
Iterations & 3 & 3 & 3 \\
Learning rate & $10^{-3}$ & $10^{-3}$ & $10^{-3}$ \\
Error tolerance & 0 & 0 & 0 \\
Training data set (FG 15/En 24) & $24 / 36$ samples & $24 / 36$ samples & $24 / 36$ samples \\
\hline
\end{tabular}



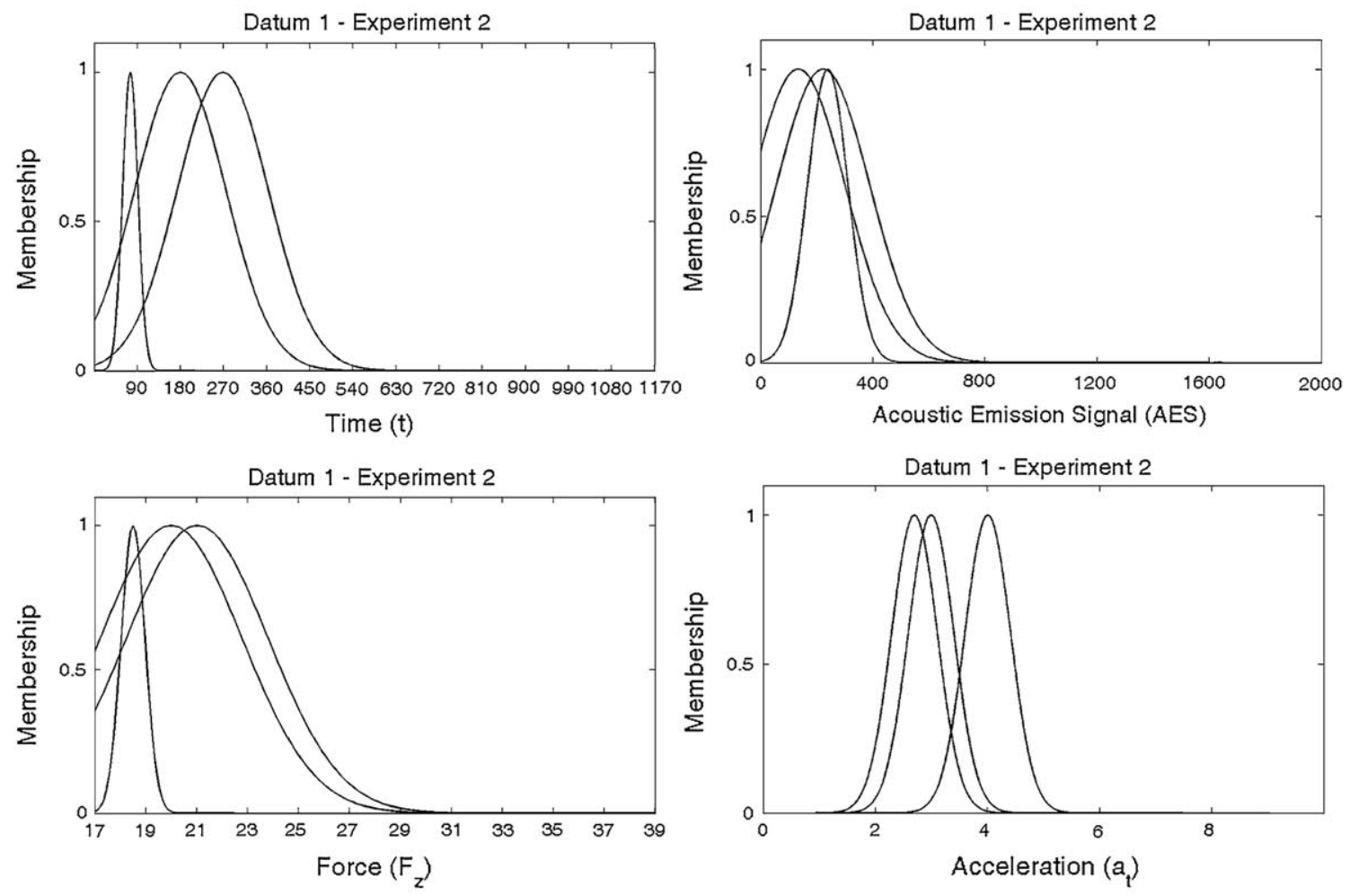

Fig. 7 Membership functions created for modelling the first element of the 4-tuple in the experiment 2

value of 0.32 . In summary the TWNFIS parameters are: four neighbors in the algorithm, a clustering threshold value of 0.32 (maximum diameter), and one as a minimum number of elements. Figure 7 shows membership functions to create the local model that corresponds to the first element of the 4-tuple $\left(t, F_{z}, a_{t}, A E S\right)$ of the experiment 2. Despite the use of four neighbors in the clustering algorithm, only 3 clusters are obtained. DENFIS parameters that best model tool wear are: 4 most strongly activated rules $(m)$, a $D_{t h r}$ of 0.01 $(\mathrm{ECM})$ and the same iterations in the training algorithm than the other neuro-fuzzy systems ( 3 iterations).

The results obtained by neuro-fuzzy models in FG 15 turning operations are shown in Fig. 8 and Table 3. TWNFIS outperformed ANFIS and DENFIS in the first and third experiment. In the second experiment, DENFIS yielded better accuracy than TWNFIS and ANFIS, and in the fourth experiment ANFIS yield the best results. However, the overall average error (4.39\%) of the TNWFIS-based model is less than the average error of ANFIS-based model (13.24\%) and DENFIS-based model (5.73\%). Likewise, it is evaluated the tool wear estimation $T_{w}^{\prime}$ greater than a threshold value of $\mathrm{AE}$ $\geq 10 \%$. The number of data points surpassing the threshold is higher in ANFIS-based model and TWNFIS-based model than in DENFIS-based model.

The results obtained by neuro-fuzzy models in En 24 turning operations are shown in Fig. 9 and Table 4. In this case, ANFIS outperformed TWNFIS and DENFIS in the first and second experiment. In the third and fourth experiment, TWNFIS yielded better accuracy than ANFIS and ADENFIS. The overall average error $(4.71 \%)$ of the TNWFIS-based model is less againt than the average error of ANFIS-based model (6.94\%) and DENFIS-based model (8.61\%). Also, the number of data points surpassing the threshold value of $\mathrm{AE}$ $\geq 10 \%$ is less in TNWFIS-based model than ANFIS-based model than in DENFIS-based model.

Therefore, TWNFIS-based model shows good overall performance in both experiments. It thus shows that the transductive neuro-fuzzy inference system is more robust against changes in cutting conditions and it is more appropiate to handle uncertainties. Nevertheless, statistical performance indices are also applied to validate the results. A modification of the Schwarz Bayesian Criterion (SBC) (14) is applied to the experiments in En 24 workpiece material (errors are closer). The results are shown in Table 5.

$$
\begin{aligned}
\text { SSE } & =\sum_{i=1}^{N}\left(T_{w}-T_{w}^{\prime}\right)^{2}(i) \\
N S S E & =\sqrt{\frac{S S E}{2 N}} \\
F P E & =\frac{N S S E(N+p)}{N-p} \\
E N V & =\frac{2 \cdot N \cdot N S S E}{N-p}
\end{aligned}
$$


Fig. 8 Real tool wear and obtained with neuro-fuzzy models for experiment 1 in FG 15 workpiece material

Table 3 Average errors of the created models (FG 15)

Fig. 9 Real tool wear and obtained with neuro-fuzzy models for experiment 6 in En 24 workpiece material

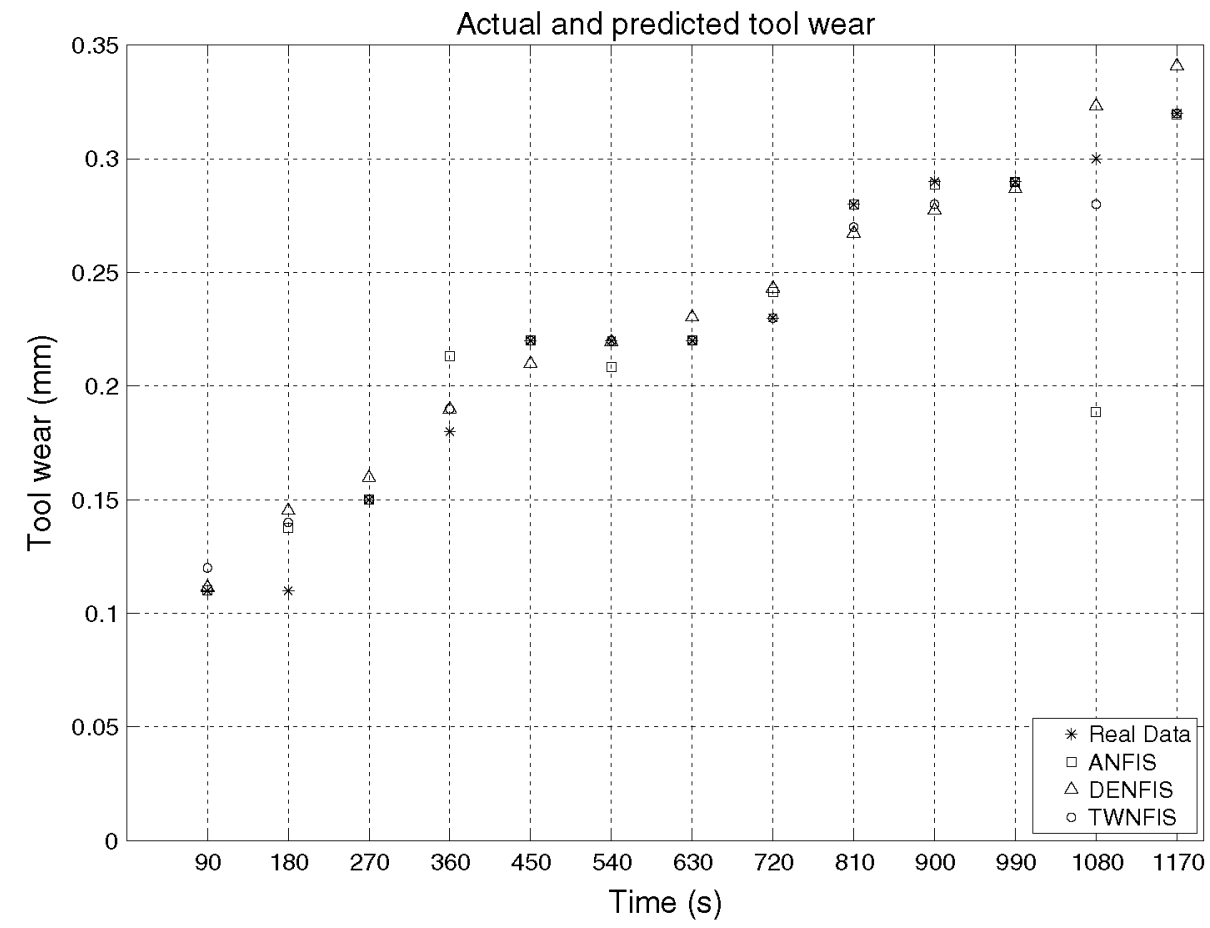

\begin{tabular}{lllllll}
\hline Experiment & $\begin{array}{l}\text { ANFIS-based } \\
\text { model }(\%)\end{array}$ & $\begin{array}{l}\text { Data points } \\
A E \geq 10 \%\end{array}$ & $\begin{array}{l}\text { DENFIS-based } \\
\operatorname{model}(\%)\end{array}$ & $\begin{array}{l}\text { Data points } \\
A E \geq 10 \%\end{array}$ & $\begin{array}{l}\text { TWNFIS-based } \\
\text { model }(\%)\end{array}$ & $\begin{array}{l}\text { Data points } \\
A E \geq 10 \%\end{array}$ \\
\hline 1 & 7.12 & 3 & 6.52 & 1 & 4.27 & 1 \\
2 & 40.40 & 4 & 4.18 & 2 & 5.04 & 3 \\
3 & 3.46 & 1 & 5.79 & 1 & 2.55 & 3 \\
4 & 1.97 & 0 & 6.46 & 1 & 5.71 & 0 \\
\hline
\end{tabular}

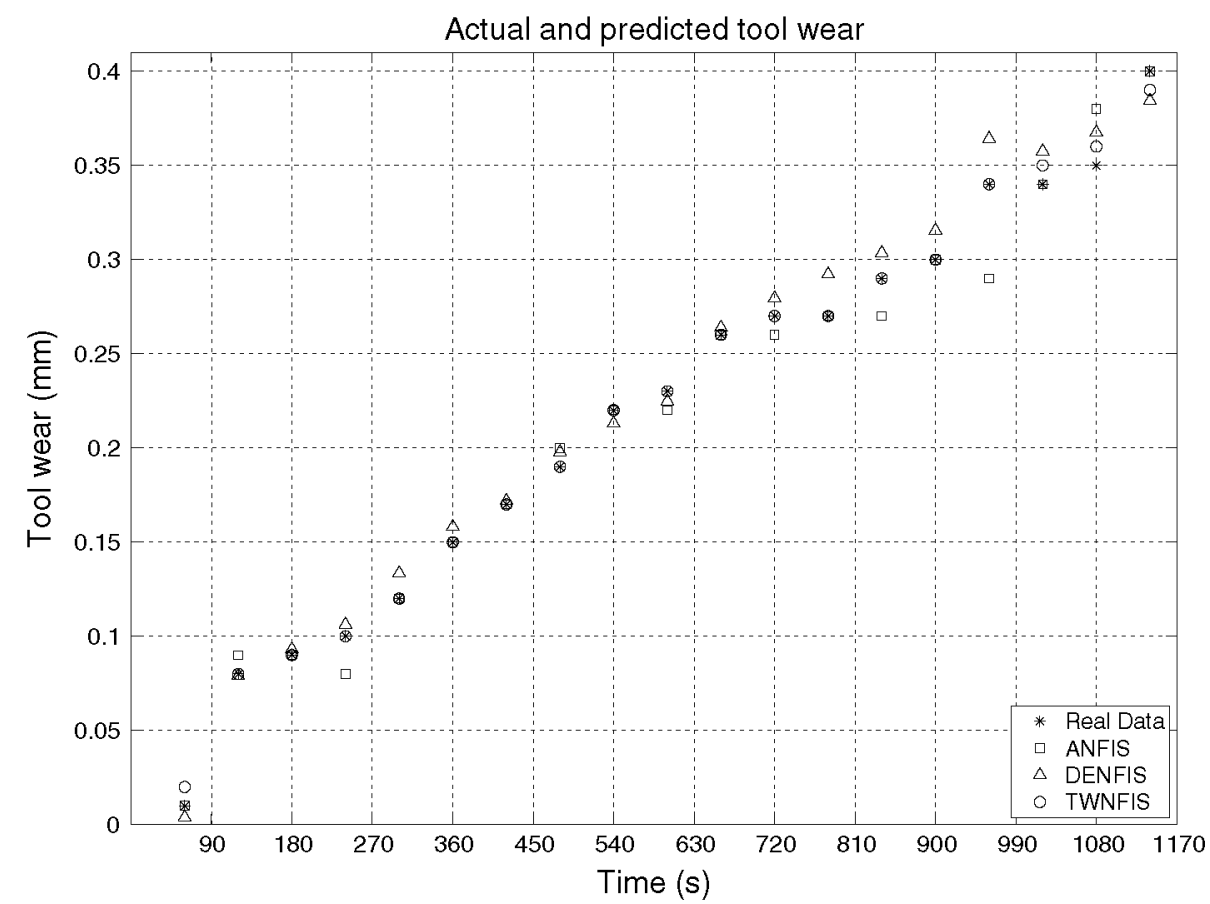


Table 4 Average errors of the created models (En 24)

\begin{tabular}{lllllll}
\hline Experiment & $\begin{array}{l}\text { ANFIS-based } \\
\text { model }(\%)\end{array}$ & $\begin{array}{l}\text { Data points } \\
A E \geq 10 \%\end{array}$ & $\begin{array}{l}\text { DENFIS-based } \\
\text { model }(\%)\end{array}$ & $\begin{array}{l}\text { Data points } \\
A E \geq 10 \%\end{array}$ & $\begin{array}{l}\text { TWNFIS-based } \\
\text { model }(\%)\end{array}$ & $\begin{array}{l}\text { Data points } \\
A E \geq 10 \%\end{array}$ \\
\hline 5 & 3.20 & 1 & 8.63 & 2 & 4.62 & 2 \\
6 & 3.99 & 2 & 7.54 & 2 & 5.70 & 1 \\
7 & 10.06 & 2 & 11.68 & 3 & 2.00 & 0 \\
8 & 10.50 & 3 & 6.59 & 2 & 6.50 & 3 \\
\hline
\end{tabular}

Table 5 Results of the modified Scharwz Bayesian Criterion (En 24)

\begin{tabular}{llll}
\hline Criterion & ANFIS & DENFIS & TWNFIS \\
\hline SSE & -0.0216 & -0.0085 & -0.0071 \\
NSSE & -0.0122 & -0.0077 & -0.0070 \\
FPE & -0.0162 & -0.0086 & -0.0078 \\
ENV & -0.0284 & -0.0163 & -0.0149 \\
SBC & -541.41 & -634.22 & -647.04 \\
\hline
\end{tabular}

Table 6 Experiment 1 (FG 15)

\begin{tabular}{lllll}
\hline$t$ & $F_{z}$ & $a_{t}$ & $A E S$ & $T_{w}$ \\
\hline 90 & 18 & 4 & 170 & $0.11^{\text {tr }}$ \\
180 & 20 & 2.5 & 179.2 & 0.11 \\
270 & 21 & 2.7 & 222 & $0.15^{\text {tr }}$ \\
360 & 23 & 2.5 & 517.8 & 0.18 \\
450 & 25 & 2.8 & 721.7 & $0.22^{\text {tr }}$ \\
540 & 25 & 2.8 & 722.8 & 0.22 \\
630 & 26 & 2.8 & 738.6 & $0.22^{\text {tr }}$ \\
720 & 27 & 3 & 745.2 & 0.23 \\
810 & 30 & 3.7 & 805.8 & $0.28^{\text {tr }}$ \\
900 & 31 & 4.2 & 845.6 & 0.29 \\
990 & 31 & 4.5 & 863.7 & $0.29^{\text {tr }}$ \\
1080 & 37 & 5 & 1128.1 & 0.3 \\
1170 & 39 & 6 & 1662.25 & $0.32^{\text {tr }}$ \\
\hline
\end{tabular}

tr Training data

$$
S B C=N \cdot \log \left(\frac{S S E}{N}\right)+p \cdot \log (N)
$$

where $S S E$ is the sum of squared errors, NSSE is the prediction error, $F P E$ is the final prediction error, $E N V$ is the estimate of the noise variance, $S B C$ is the modification of the Scharwz Bayesian Criterion, $p$ is the number of rules and $N$ is the total number of samples. The results of the application of this criterion show again that TWNFIS is the most suitable neuro-fuzzy system for modeling and monitoring the tool wear of turning processes (Tables 6, 7, 8, 9, 10, 11, 12, $13)$.
Table 7 Experiment 2 (FG 15)

\begin{tabular}{|c|c|c|c|c|}
\hline$t$ & $F_{z}$ & $a_{t}$ & $A E S$ & $T_{w}$ \\
\hline 90 & 19 & 4 & 114.4 & 0.12 \\
\hline 180 & 20 & 3 & 137 & $0.13^{\text {tr }}$ \\
\hline 270 & 22 & 2 & 190.7 & 0.15 \\
\hline 360 & 22 & 3 & 238 & $0.17^{\mathrm{tr}}$ \\
\hline 450 & 23 & 2 & 334.6 & 0.19 \\
\hline 540 & 25 & 3 & 616.6 & $0.22^{\mathrm{tr}}$ \\
\hline 630 & 26 & 3 & 654.8 & 0.23 \\
\hline 720 & 27 & 3.5 & 667.4 & $0.23^{\text {tr }}$ \\
\hline 810 & 30 & 4 & 699 & 0.24 \\
\hline 900 & 32 & 5 & 865.5 & $0.27^{\mathrm{tr}}$ \\
\hline 990 & 37 & 7 & 1302 & 0.3 \\
\hline 1080 & 39 & 8 & 1526.2 & $0.32^{\mathrm{tr}}$ \\
\hline 1170 & 48 & 9 & 2545.5 & 0.37 \\
\hline \multicolumn{5}{|c|}{ tr Training data } \\
\hline \multicolumn{5}{|c|}{ Table 8 Experiment 3 (FG 15) } \\
\hline$t$ & $F_{z}$ & $a_{t}$ & $A E S$ & $T_{w}$ \\
\hline 60 & 19 & 4 & 308.6 & $0.12^{\mathrm{tr}}$ \\
\hline 120 & 20 & 2.5 & 564.4 & 0.15 \\
\hline 180 & 21 & 2 & 742 & $0.17^{\mathrm{tr}}$ \\
\hline 240 & 23 & 2.5 & 849.6 & 0.19 \\
\hline 300 & 26 & 2 & 976.6 & $0.22^{\text {tr }}$ \\
\hline 360 & 29 & 5.5 & 1006 & 0.24 \\
\hline 420 & 30 & 2.5 & 1067 & $0.25^{\text {tr }}$ \\
\hline 480 & 31 & 4 & 1086.4 & 0.26 \\
\hline 540 & 33 & 3.9 & 1138.4 & $0.28^{\mathrm{tr}}$ \\
\hline 600 & 42 & 4.2 & 1576.6 & 0.36 \\
\hline 660 & 52 & 5 & 1992.4 & $0.4^{\text {tr }}$ \\
\hline
\end{tabular}

tr Training data

\section{Conclusions}

This paper presents two methods for tool wear monitoring in turning processes, based on neuro-fuzzy models. A fourinput (time, cutting forces, vibrations and acoustic emissions signals) single-output (tool wear rate) model has been implemented on the basis of different neuro-fuzzy approaches 
Table 9 Experiment 4 (FG 15)

\begin{tabular}{lllll}
\hline$t$ & $F_{z}$ & $a_{t}$ & $A E S$ & $T_{w}$ \\
\hline 60 & 20 & 3.8 & 1046.3 & 0.14 \\
120 & 20 & 3 & 1054.2 & $0.14^{\text {tr }}$ \\
180 & 22 & 2.5 & 1072.5 & 0.15 \\
240 & 22 & 2.5 & 1092 & $0.15^{\text {tr }}$ \\
300 & 28 & 2.8 & 1301 & 0.22 \\
360 & 32 & 3 & 1443 & $0.25^{\text {tr }}$ \\
420 & 34 & 3.2 & 1644.5 & 0.29 \\
480 & 35 & 3.5 & 1645.7 & $0.3^{\text {tr }}$ \\
540 & 38 & 4 & 1668 & 0.32 \\
600 & 52 & 4 & 2058.7 & $0.4^{\text {tr }}$ \\
\hline
\end{tabular}

tr Training data

Table 10 Experiment 5 (En 24)

\begin{tabular}{lllll}
\hline$t$ & $F_{z}$ & $a_{t}$ & $A E S$ & $T_{w}$ \\
\hline 60 & 3 & 0.08 & 593.8 & 0.02 \\
120 & 9 & 0.1 & 1387.3 & $0.08^{\text {tr }}$ \\
180 & 14 & 0.12 & 4354.5 & 0.15 \\
240 & 15 & 0.13 & 4652 & $0.16^{\text {tr }}$ \\
300 & 16 & 0.13 & 4728.1 & 0.17 \\
360 & 17 & 0.12 & 5079.8 & $0.19^{\text {tr }}$ \\
420 & 18 & 0.08 & 5248 & 0.2 \\
480 & 18 & 0.08 & 5262.1 & $0.2^{\text {tr }}$ \\
540 & 19 & 0.07 & 5318.8 & 0.21 \\
600 & 19 & 0.08 & 5370.6 & $0.21^{\text {tr }}$ \\
660 & 20 & 0.08 & 5417.1 & 0.22 \\
720 & 21 & 0.09 & 5470.6 & $0.23^{\text {tr }}$ \\
780 & 21 & 0.09 & 5524.8 & 0.23 \\
840 & 21 & 0.08 & 5533.3 & $0.23^{\text {tr }}$ \\
900 & 22 & 0.1 & 5594.6 & 0.24 \\
960 & 23 & 0.11 & 5763.5 & $0.26^{\text {tr }}$ \\
1020 & 24 & 0.12 & 5886.8 & 0.27 \\
1080 & 29 & 0.15 & 6012.5 & $0.34^{\text {tr }}$ \\
1140 & 30 & 0.16 & 6462.3 & 0.35 \\
\hline & & & &
\end{tabular}

tr Training data

(inductive, transductive and evolving neuro-fuzzy systems). The obtained results demonstrate that the proposed neurofuzzy systems (ANFIS, DENFIS and TWNFIS) are capable of dealing with the non-linearity and the uncertainties of the tool wear process.

Moreover, this paper reports the first application of the Dynamic Evolving Neural-Fuzzy Inference System (DENFIS) and of the Transductive-Weighted Neuro-Fuzzy Inference System (TWNFIS) to the tool wear modeling problem. The results of the comparative study shows the superiority of TWNFIS: it provides smaller errors than DENFIS and ANFIS, and it does with less rules than the others. Although
Table 11 Experiment 6 (En 24)

\begin{tabular}{lllll}
\hline$t$ & $F_{z}$ & $a_{t}$ & $A E S$ & $T_{w}$ \\
\hline 60 & 4 & 0.08 & 427 & $0.01^{\text {tr }}$ \\
120 & 10 & 0.09 & 1787.1 & 0.08 \\
180 & 11 & 0.1 & 1949.1 & $0.09^{\text {tr }}$ \\
240 & 12 & 0.1 & 2166 & 0.1 \\
300 & 14 & 0.11 & 2474 & $0.12^{\text {tr }}$ \\
360 & 16 & 0.12 & 2677.1 & 0.15 \\
420 & 17 & 0.13 & 2965.6 & $0.17^{\text {tr }}$ \\
480 & 19 & 0.15 & 3236.3 & 0.19 \\
540 & 20 & 0.17 & 3786 & $0.22^{\text {tr }}$ \\
600 & 21 & 0.18 & 4063.1 & 0.23 \\
660 & 24 & 0.2 & 4837.1 & $0.26^{\text {tr }}$ \\
720 & 25 & 0.21 & 5237.5 & 0.27 \\
780 & 26 & 0.22 & 5245.1 & $0.27^{\text {tr }}$ \\
840 & 27 & 0.24 & 5502.3 & 0.29 \\
900 & 28 & 0.25 & 5746.6 & $0.3^{\text {tr }}$ \\
960 & 31 & 0.26 & 7074.5 & 0.34 \\
1020 & 31 & 0.28 & 7070 & $0.34^{\text {tr }}$ \\
1080 & 32 & 0.29 & 7111.5 & 0.35 \\
1140 & 33 & 0.3 & 7856.5 & $0.4^{\text {tr }}$ \\
\hline & & & &
\end{tabular}

tr Training data

Table 12 Experiment 7 (En 24)

\begin{tabular}{lllll}
\hline$t$ & $F_{z}$ & $a_{t}$ & $A E S$ & $T_{w}$ \\
\hline 60 & 4 & 0.09 & 21.5 & 0.02 \\
120 & 8 & 0.09 & 22.1 & $0.03^{\text {tr }}$ \\
180 & 12 & 0.13 & 46 & 0.1 \\
240 & 13 & 0.13 & 48.8 & $0.11^{\text {tr }}$ \\
300 & 15 & 0.15 & 63.5 & 0.13 \\
360 & 17 & 0.08 & 86 & $0.15^{\text {tr }}$ \\
420 & 17 & 0.08 & 89.3 & 0.15 \\
480 & 18 & 0.09 & 92.8 & $0.16^{\text {tr }}$ \\
540 & 18 & 0.1 & 92.6 & 0.16 \\
600 & 19 & 0.11 & 98.5 & $0.17^{\text {tr }}$ \\
660 & 22 & 0.15 & 109 & 0.2 \\
720 & 29 & 0.17 & 168.1 & $0.3^{\text {tr }}$ \\
780 & 30 & 0.18 & 170 & 0.31 \\
840 & 31 & 0.19 & 179.6 & $0.32^{\text {tr }}$ \\
900 & 32 & 0.21 & 187.3 & 0.33 \\
960 & 32 & 0.22 & 350.5 & $0.38^{\text {tr }}$ \\
1020 & 33 & 0.25 & 462.1 & 0.41 \\
\hline
\end{tabular}

tr Training data

previous work (Gajate et al. 2009) achieved promising initial results, this study has shown that TWNFIS is completely valid for turning operations with other workpiece materials. The fact is that the transductive inference provides greater 
Table 13 Experiment 8 (En 24)

\begin{tabular}{lllll}
\hline$t$ & $F_{z}$ & $a_{t}$ & $A E S$ & $T_{w}$ \\
\hline 60 & 4 & 0.13 & 13.6 & $0.01^{\mathrm{tr}}$ \\
120 & 9 & 0.2 & 99.5 & 0.06 \\
180 & 10 & 0.2 & 129.6 & $0.08^{\mathrm{tr}}$ \\
240 & 12 & 0.25 & 229.6 & 0.12 \\
300 & 15 & 0.18 & 327 & $0.15^{\mathrm{tr}}$ \\
360 & 18 & 0.12 & 716.8 & 0.18 \\
420 & 19 & 0.12 & 791.7 & $0.19^{\mathrm{tr}}$ \\
480 & 21 & 0.2 & 902 & 0.21 \\
540 & 25 & 0.24 & 3389.8 & $0.3^{\mathrm{tr}}$ \\
600 & 26 & 0.24 & 3911 & 0.31 \\
660 & 30 & 0.25 & 4720.1 & $0.35^{\mathrm{tr}}$ \\
720 & 33 & 0.26 & 5592.1 & 0.39 \\
780 & 35 & 0.28 & 6177.1 & $0.42^{\mathrm{tr}}$ \\
840 & 37 & 0.3 & 6452.6 & 0.45 \\
900 & 38 & 0.31 & 6510.4 & $0.46^{\mathrm{tr}}$ \\
960 & 40 & 0.32 & 7693.2 & 0.5 \\
1020 & 41 & 0.33 & 7935 & $0.52^{\mathrm{tr}}$ \\
\hline
\end{tabular}

tr Training data

benefits than the rest of inductive inferences in the case of monitoring tool wear. Therefore, in this case study, is better to use local models because they represent the behavior better than general models.

Future work will address the use fuzzy clustering in TWNFIS and the application to other modeling problems.

Acknowledgments This work was supported by DPI2008-01978 "Networked cognitive control system for high-performance machining processes (COGNETCON)" and CIT-420000-2008-13 "Intelligent monitoring of nano-scale cutting processes in manufacturing complex aeronautical parts (NANOCUT-INT)" projects of the Spanish Ministry of Science and Innovation.

\section{References}

Abellan-Nebot, J. V., \& Romero Subiron, F. (2010). A review of machining monitoring systems based on artificial intelligence process models. International Journal of Advanced Manufacturing Technology, 47, 237-257.

Cakmakci, M., Kinaci, C., Bayramoglu, M., \& Yildirim, Y. (2010). A modeling approach for iron concentration in sand filtration effluent using adaptive neuro-fuzzy model. Expert Systems with Applications, 37(2), 1369-1373.

Denai, M. A., Palis, F., \& Zeghbib, A. (2007). Modeling and control of non-linear systems using soft computing techniques. Applied Soft Computing Journal, 7(3), 728-738.

Dimla, D. (2000). Sensor signals for tool-wear monitoring in metal cutting operations-A review of methods. International Journal of Machine Tools and Manufacture, 40(8), 1073-1098.

Dinakaran, D., Sampathkumar, S., \& Sivashanmugam, N. (2009). An experimental investigation on monitoring of crater wear in turning using ultrasonic technique. International Journal of Machine Tools and Manufacture, 49(15), 1234-1237.

Gajate, A., Haber, R. E., Alique, J. R., \& Vega, P. I. (2009). Transductive-weighted neuro-fuzzy inference system for tool wear prediction in a turning process. Lecture Notes in Artificial Intelligence (Vol. 5572, pp. 113-120).

Hayati, M., Rezaei, A., \& Seif, M. (2009). Prediction of the heat transfer rate of a single layer wire-on-tube type heat exchanger using ANFIS. International Journal of Refrigeration, 32(8), 1914-1917.

Heyer, L., Kruglyak, S., \& Yooseph, S. (1999). Exploring expression data: Identification and analysis of coexpressed genes. Genome Research, 9(11), 1106-1115.

Jang, J. (1993). ANFIS: Adaptive-network-based fuzzy inference system. IEEE Transactions on Systems, Man, and Cybernetics, 23(3), 665-685.

Kasabov, N., \& Song, Q. (2002). Denfis: Dynamic evolving neural-fuzzy inference system and its application for time-series prediction. IEEE Transactions on Fuzzy Systems, 10(2), 144-154.

Li, X., Djordjevich, A., \& Venuvinod, P. K. (2000). Current-sensor-based feed cutting force intelligent estimation and tool wear condition monitoring. IEEE Transactions on Industrial Electronics, 47(3), 697-702.

Li, X., Li, H. X., Guan, X. P., \& Du, R. (2004). Fuzzy estimation of feed-cutting force from current measurement-a case study on intelligent tool wear condition monitoring. IEEE Transactions on Systems, Man and Cybernetics Part C: Applications and Reviews, 34(4), 506-512.

Liang, S. Y., Hecker, R. L., \& Landers, R. G. (2004). Machining process monitoring and control: The state-of-the-art. Journal of Manufacturing Science and Engineering, Transactions of the ASME, 126(2), 297-310.

Pal, S., Heyns, P. S., Freyer, B. H., Theron, N. J., \& Pal, S. K. (2009). Tool wear monitoring and selection of optimum cutting conditions with progressive tool wear effect and input uncertainties. Journal of Intelligent Manufacturing (in press).

Perez, J. A., Gonzalez, M., \& Dopico, D. (2010). Adaptive neurofuzzy ANFIS modeling of laser surface treatments. Neural Computing and Applications, 19(1), 85-90.

Purushothaman, S. (2009). Tool wear monitoring using artificial neural network based on extended Kalman filter weight updation with transformed input patterns. Journal of Intelligent Manufacturing (in press).

Rehorn, A. G., Jiang, J., \& Orban, P. E. (2005). State-of-the-art methods and results in tool condition monitoring: A review. International Journal of Advanced Manufacturing Technology, 26(7-8), $693-710$.

Rubio, E. M., \& Teti, R. (2009). Cutting parameters analysis for the development of a milling process monitoring system based on audible energy sound. Journal of Intelligent Manufacturing, 20(1), 43-54.

Sargolzaei, J., \& Kianifar, A. (2010). Neuro-fuzzy modeling tools for estimation of torque in Savonius rotor wind turbine. Advances in Engineering Software, 41(4), 619-626.

Sharma, V., Sharma, S., \& Sharma, A. (2008a). Cutting tool wear estimation for turning. Journal of Intelligent Manufacturing, 19(1), 99-108.

Sharma, V. S., Sharma, S. K., \& Sharma, A. K. (2007). An approach for condition monitoring of a turning tool. Proceedings of the Institution of Mechanical Engineers, Part B: Joumal of Engineering Manufacture, 227(4), 635-646.

Sharma, V. S., Dogra, M., \& Suri, N. M. (2008b). Advances in the turning process for productivity improvement-A review. Proceedings of the Institution of Mechanical Engineers, Part B: Journal of Engineering Manufacture, 222(11), 1417-1442.

Sick, B. (2002). On-line and indirect tool wear monitoring in turning with artificial neural networks: A review of more than a decade of 
research. Mechanical Systems and Signal Processing, 16(4), 487546.

Sjoberg, J., Zhang, Q., Ljung, L., Benveniste, A., Delyon, B., Glorennec, P., Hjalmarsson, H., \& Juditsky, A. (1995). Nonlinear black-box modeling in system identification: A unified overview. Automatica, 31(12), 1691-1724.

Song, Q., \& Kasabov, N. (2006). TWNFI: A transductive neurofuzzy inference system with weighted data normalization for personalized modeling. Neural Networks, 19(10), 1591-1596.

Song, Q., \& Kasasbov, N. (2001). ECM, a novel on-line, evolving clustering method and its applications. In Proceedings of 5th Biannu Conf Artif Neural Netw Expert Syst-ANNES 2001 (pp 87-92).
Ubeyli, E. D. (2009). Adaptive neuro-fuzzy inference systems for automatic detection of breast cancer. Journal of Medical Systems, 33(5), 353-358.

Wang, X., Wang, W., Huang, Y., Nguyen, N., \& Krishnakumar, K. (2008). Design of neural network-based estimator for tool wear modeling in hard turning. Journal of Intelligent Manufacturing, 19(4), 383-396.

Warnecke, G., \& Kluge, R. (1998). Control of tolerances in turning by predictive control with neural networks. Journal of Intelligent Manufacturing, 9(4), 281-287.

Watts, M. J. (2009). A decade of Kasabov's evolving connectionist systems: A review. IEEE Transactions on Systems, Man and Cybernetics Part C: Applications and Reviews, 39(3), 253-269. 